\title{
Nano-Ag on vanadium dioxide. II. Thermal tuning of surface plasmon resonance
}

\author{
Gang Xu, ${ }^{1, a)}$ Chun-Ming Huang, ${ }^{1}$ Masato Tazawa, ${ }^{2, b)}$ Ping Jin, ${ }^{2}$ and De-Ming Chen ${ }^{1}$ \\ ${ }^{1}$ Key Laboratory of Renewable Energy and Gas Hydrate, Guangzhou Institute of Energy Conversion, \\ Chinese Academy of Sciences, Guangzhou 510640, People's Republic of China \\ ${ }^{2}$ National Institute of Advanced Industrial Science and Technology, Nagoya 463-8560, Japan
}

(Received 13 May 2008; accepted 24 July 2008; published online 2 September 2008)

\begin{abstract}
Thermal tuning of the localized surface plasmon resonance (LSPR) of Ag nanoparticles on a thermochromic thin film of $\mathrm{VO}_{2}$ was studied experimentally. The tuning is strongly temperature dependent and thermally reversible. The LSPR wavelength $\lambda_{\text {SPR }}$ shifts to the blue with increasing temperature from 30 to $80{ }^{\circ} \mathrm{C}$, and shifts back to the red as temperature decreases. A smart tuning is achievable on condition that the temperature is controlled in a stepwise manner. The tunable wavelength range depends on the particle size or the mass thickness of the metal nanoparticle film. Further, the tunability was found to be enhanced significantly when a layer of $\mathrm{TiO}_{2}$ was introduced to overcoat the Ag nanoparticles, yielding a marked sensitivity factor $\Delta \lambda_{\mathrm{SPR}} / \Delta n$, of as large as 480 $\mathrm{nm}$ per refractive index unit $(n)$ at the semiconductor phase of $\mathrm{VO}_{2}$. (C) 2008 American Institute of Physics. [DOI: 10.1063/1.2973341]
\end{abstract}

\section{INTRODUCTION}

Noble metal nanoparticles have attracted a considerable interest for their unique optical properties characterized by localized surface plasmon resonance (LSPR). Generally, LSPR is excited when the incident light with a specific wavelength interacts with the metal nanoparticles to create the collective oscillation of conduction electrons. This LSPR is accompanied by wavelength-selective photon absorption and resonant Mie scattering and by a dramatic enhancement of the local electromagnetic field in the vicinity of the nanoparticles. ${ }^{1-3}$ Such unique optical properties make the metal nanoparticle extremely valuable in a variety of technological applications such as plasmon-based optical devices, ${ }^{4}$ chemical and biological sensors, ${ }^{5,6}$ and surface-enhanced spectroscopies. $^{7,8}$

Most of the above mentioned applications require the LSPR wavelength $\left(\lambda_{\mathrm{SPR}}\right)$ tunable over a wide spectral range. In view of the fact that $\lambda_{\text {SPR }}$ depends on the size, shape, and composition of the metal nanoparticles, as well as on the surrounding dielectric environment, ${ }^{2-4}$ the tunable wavelength range from visible to near infrared (NIR) regions has been achieved by varying these parameters. ${ }^{3,9-12}$ However, almost all of those tuning methods are independent of temperature since the resonance of noble metal nanoparticle is known to be an electronic mechanism and insensitive to temperature. ${ }^{13-16}$ It has been demonstrated that there was little change in the $\lambda_{\mathrm{SPR}}$ of Ag nanoparticle when the particles were heated from room temperature up to $500{ }^{\circ} \mathrm{C} .{ }^{13}$ Such a behavior of the noble metal nanoparticles may hinder their applications in temperature-sensitive optical devices or sensors. ${ }^{6,17}$

On the other hand, $\mathrm{VO}_{2}$ is a well-known thermochromic material. It undergoes a reversible first-order semiconductor-

\footnotetext{
a)Electronic mail: xugang@ms.giec.ac.cn.

${ }^{b)}$ Electronic mail: tazawa.m@aist.go.jp.
}

metal transition (SMT) at critical temperature $t_{c} \approx 68{ }^{\circ} \mathrm{C}$, resulting in dramatic change in electrical and optical properties. ${ }^{18-20}$ Of particular interest is the optical change through the SMT in the IR region where $\mathrm{VO}_{2}$ varies from being transparent in the semiconductor phase to highly reflective in the metal phase, implying that the refractive index of $\mathrm{VO}_{2}$ changes with temperature. ${ }^{20}$ Hence, thermal tuning of the resonance of the metal nanoparticles can be achieved by taking advantage of this optical change in $\mathrm{VO}_{2}$ stimulated by temperature.

Recently, such a thermal tuning of the resonance has been demonstrated. ${ }^{21-25} \mathrm{Xu}$ et al. $^{21}$ reported the temperature tunability of $\lambda_{\mathrm{SPR}}$ of $\mathrm{Ag}$ nanoparticle on $\mathrm{VO}_{2}$ substrate. Also, a similar study was carried out by Maaza et al. ${ }^{23,24}$ with $\mathrm{Au}$ nanoparticles dispersed in the $\mathrm{VO}_{2}$ matrix.

Following our previous study mentioned above, in this work we investigate in detail how the $\lambda_{\text {SPR }}$ of Ag nanoparticle is affected by SMT of $\mathrm{VO}_{2}$. It demonstrates that $\lambda_{\mathrm{SPR}}$ is strongly temperature dependent and reversibly tunable. A smart tuning of $\lambda_{\mathrm{SPR}}$ can be achieved by controlling the external temperature in a stepwise manner. The tuning wavelength range is highly correlated with the particle size or the mass thickness of the nano-Ag film. A high-index dielectric medium $\mathrm{TiO}_{2}$ was introduced to overcoat the Ag nanoparticles for both wavelength tuning and protecting Ag surface from photochemical degradation by absorbed species. Strikingly, it reveals that upon addition of $\mathrm{TiO}_{2}$ the wavelength tunability of nano- $\mathrm{Ag}$ on $\mathrm{VO}_{2}$ is enhanced significantly.

\section{EXPERIMENTS}

The fabrication methods for the two series of composite films of nano- $\mathrm{Ag} / \mathrm{VO}_{2}$ and $\mathrm{TiO}_{2} /$ nano- $\mathrm{Ag} / \mathrm{VO}_{2}$ with various Ag mass thicknesses were described in the prior Paper. ${ }^{26}$ The thickness of the $\mathrm{VO}_{2}$ layer is $\sim 50 \mathrm{~nm}$. For comparison, a Ag nanoparticle film with mass thickness of $10 \mathrm{~nm}$ was also deposited onto a bare sapphire slide, which is referred to as 

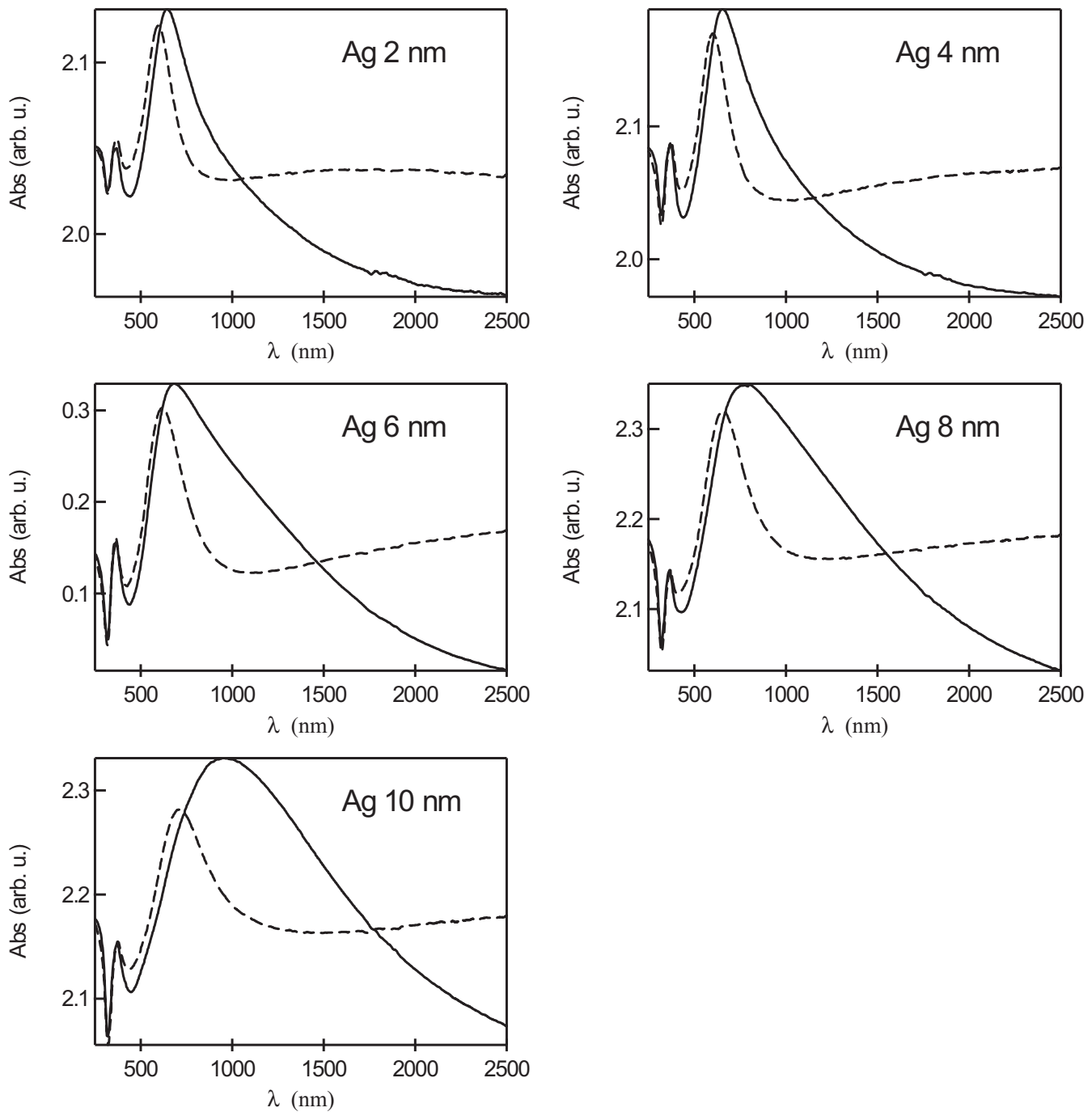

FIG. 1. Absorbance spectra of the AVS samples with various Ag mass thicknesses for both of the semiconductor (solid curves) and the metal (dashed curves) phases.

AS. The samples with a layer configuration of nano- $\mathrm{Ag} /$ quartz, nano- $\mathrm{Ag} / \mathrm{VO}_{2} /$ sapphire, and $\mathrm{TiO}_{2} /$ nano- $\mathrm{Ag} /$ $\mathrm{VO}_{2} /$ sapphire are named as AQ, AVS, and TAVS, respectively.

Atomic force microscopy images of these samples were collected on a Nanoscope III scanning probe microscope (Digital Instruments) operated in tapping mode and the results were presented in the prior Paper. ${ }^{26}$ Absorbance spectra of these samples were recorded on a UV-vis-NIR spectrophotometer (V-570, Jasco) with an attachment to control the external temperature of the samples. A silver-free $\mathrm{VO}_{2}$-coated sapphire substrate was used as the reference during the measurements.

\section{RESULTS AND DISCUSSION}

Figure 1 shows the absorbance spectra of AVS samples with various $\mathrm{Ag}$ mass thicknesses $\left(d_{m}=2,4,6,8\right.$, and 10 $\mathrm{nm})$. The solid curves in this figure correspond to the semiconductor phase of $\mathrm{VO}_{2}$ measured at $30^{\circ} \mathrm{C}$ and the dashed curves correspond to the metal phase at $80^{\circ} \mathrm{C}$. It can be seen in the figure that the $\lambda_{\mathrm{SPR}}$ of $\mathrm{Ag}$ nanoparticles shifts to the blue when temperature increases from 30 to $80{ }^{\circ} \mathrm{C}$ for all of the five samples. It is well established that the SPR of noble metallic particles only shows a very small temperature effect due to the fact that the dominant electronic dephasing mechanism involves electron-electron interactions rather than electron-phonon coupling. ${ }^{14-16}$ Thus, it is believed that the significant shift of $\lambda_{\text {SPR }}$ originates from the change in dielectric constants of the supporting material $\mathrm{VO}_{2}$.

The influence of Ag mass thickness $d_{m}$ on $\lambda_{\text {SPR }}$ seems to be very pronounced, as shown in Fig. 1 . An increase in $d_{m}$ not only leads to a redshift of $\lambda_{\text {SPR }}$ for both of the semiconductor and metal phases but also enlarges the difference in $\lambda_{\text {SPR }}$, namely, $\Delta \lambda_{\text {SPR }}$ between the two phases. For the case of $d_{m}=2 \mathrm{~nm}$, for example, the SPR wavelength $\lambda_{\mathrm{SPR}}$ locates at $650 \mathrm{~nm}$ for the semiconductor phase and at $600 \mathrm{~nm}$ for the metal phase, with a difference $\Delta \lambda_{\mathrm{SPR}}=50 \mathrm{~nm}$. In contrast, for the case of $d_{m}=8 \mathrm{~nm}, \lambda_{\mathrm{SPR}}$ locates at 780 and $660 \mathrm{~nm}$ for the two phases, respectively, with $\Delta \lambda_{\mathrm{SPR}}=120 \mathrm{~nm}$. When $d_{m}$ increases further, $\lambda_{\text {SPR }}$ redshifts more, and $\Delta \lambda_{\text {SPR }}$ becomes much larger as well with the largest value of $\Delta \lambda_{\text {SPR }}$ being $250 \mathrm{~nm}$ obtained for $d_{m}=10 \mathrm{~nm}$. The redshift of $\lambda_{\text {SPR }}$ with increasing $d_{m}$ is attributed to the dipole-dipole interaction of the particles. ${ }^{1}$ Such interaction becomes more significant if 

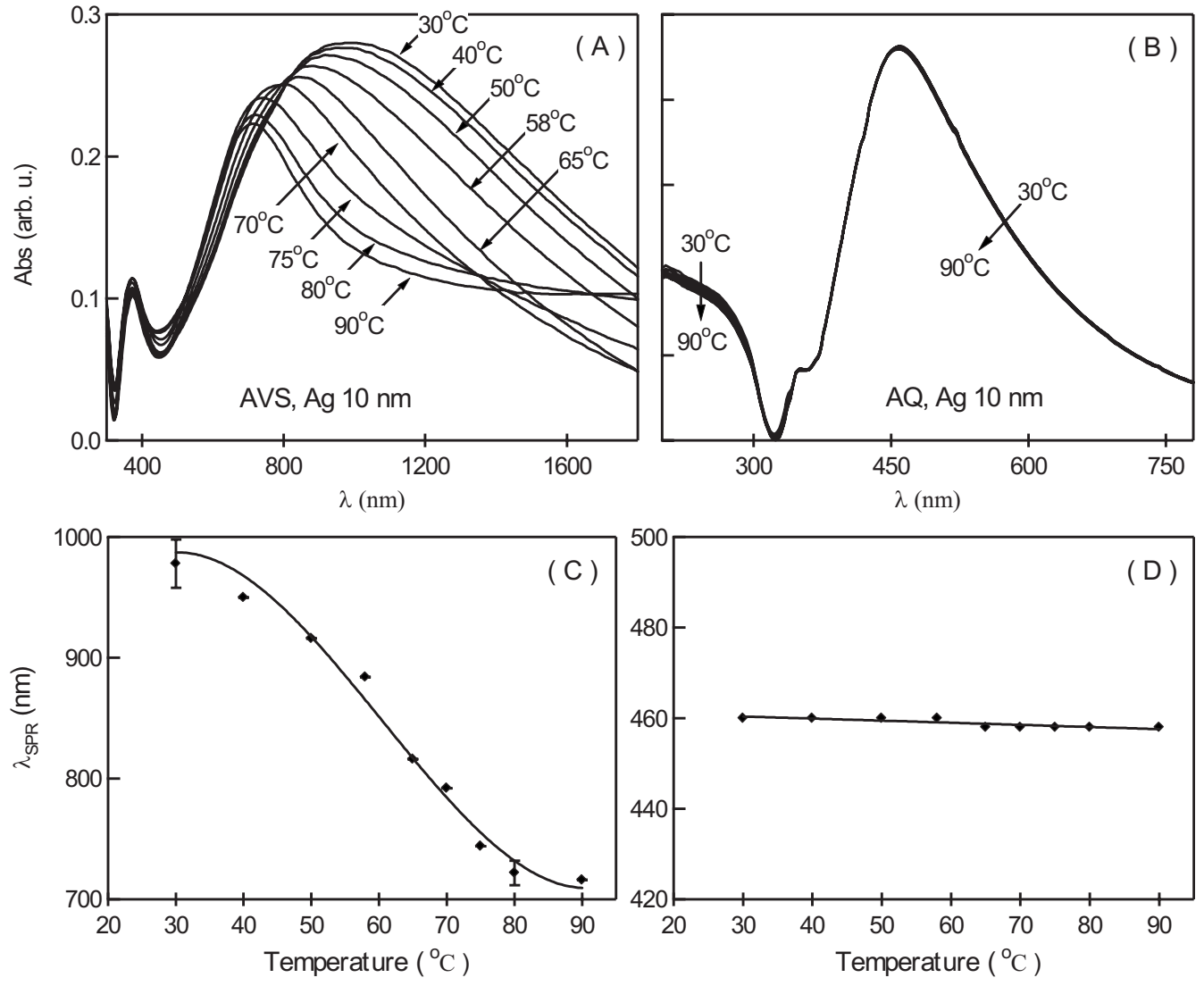

FIG. 2. Tuning of the LSPR band for (a) the AVS and (b) the AQ samples by increasing temperature from 30 to $90{ }^{\circ} \mathrm{C}$ in a stepwise manner. The resonance wavelength shifts with temperature in case of (c) the AVS and (d) the AQ samples. The Ag mass thickness is $10 \mathrm{~nm}$.

the particles become larger and the interparticle spacing becomes shorter, which is the case for a natural growth of metal nanoparticles, e.g., thermal evaporation or sputtering, when metal mass thickness inceases. ${ }^{21}$ The enlargement in $\Delta \lambda_{\text {SPR }}$ results from the increasing difference in dielectric function between the two phases of $\mathrm{VO}_{2}$ as wavelength increases. ${ }^{21}$

It is worthy to note in Fig. 1 that a subpeak located at around $390 \mathrm{~nm}$ is always present in all of the absorbance spectra. Its position is fixed neither shifting with temperature nor with Ag mass thickness. This is quite distinct from resonance properties of noble metal nanoparticles contributed by dipole and/or quadrupole interactions. Additionally, it can be seen that the LSPR absorbance band in the IR region for the metal phase has an upward tail. Although the spectrum of the $\mathrm{VO}_{2}$ / sapphire substrate was taken as a reference during the measurement, we believe that all of these spectral features are caused by the supporting material $\left(\mathrm{VO}_{2}\right)$ of the particles. A similar phenomenon was observed when $\mathrm{Ag}$ nanoparticles were deposited on indium tin oxide-coated glass. ${ }^{27}$ Based on effective medium theory, we demonstrated that the dielectric properties of the substrates have marked effects on the spectral line shape of LSPR; beyond the plasmon resonance band, the spectral shape is predominantly governed by the dielectric function, particularly its imaginary part, of the substrate. This implies that the effect of the substrate cannot be excluded even when using a substrate as the reference, in particular the substrate being strongly absorbing. Actually, it is easy to find that the imaginary part $\varepsilon_{2}(\lambda)$ (Ref. 21) of the
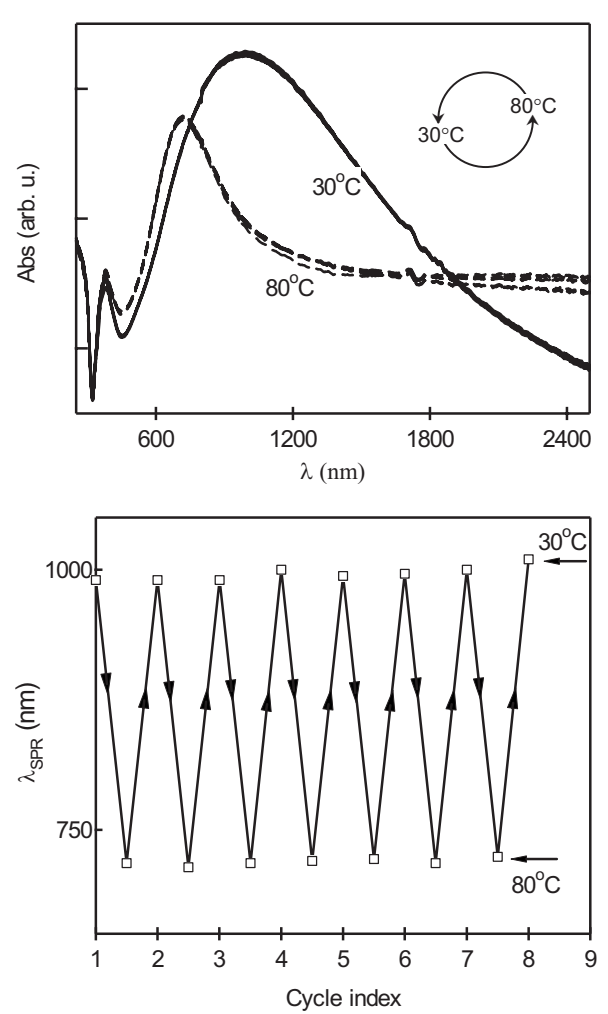

FIG. 3. Absorbance spectra of an AVS sample $\left(d_{m}=10 \mathrm{~nm}\right)$ measured at 30 and $80{ }^{\circ} \mathrm{C}$ under the repetitions of heating-cooling cycle (upper part) and the corresponding location of the resonance wavelength at each cycle (bottom part). 

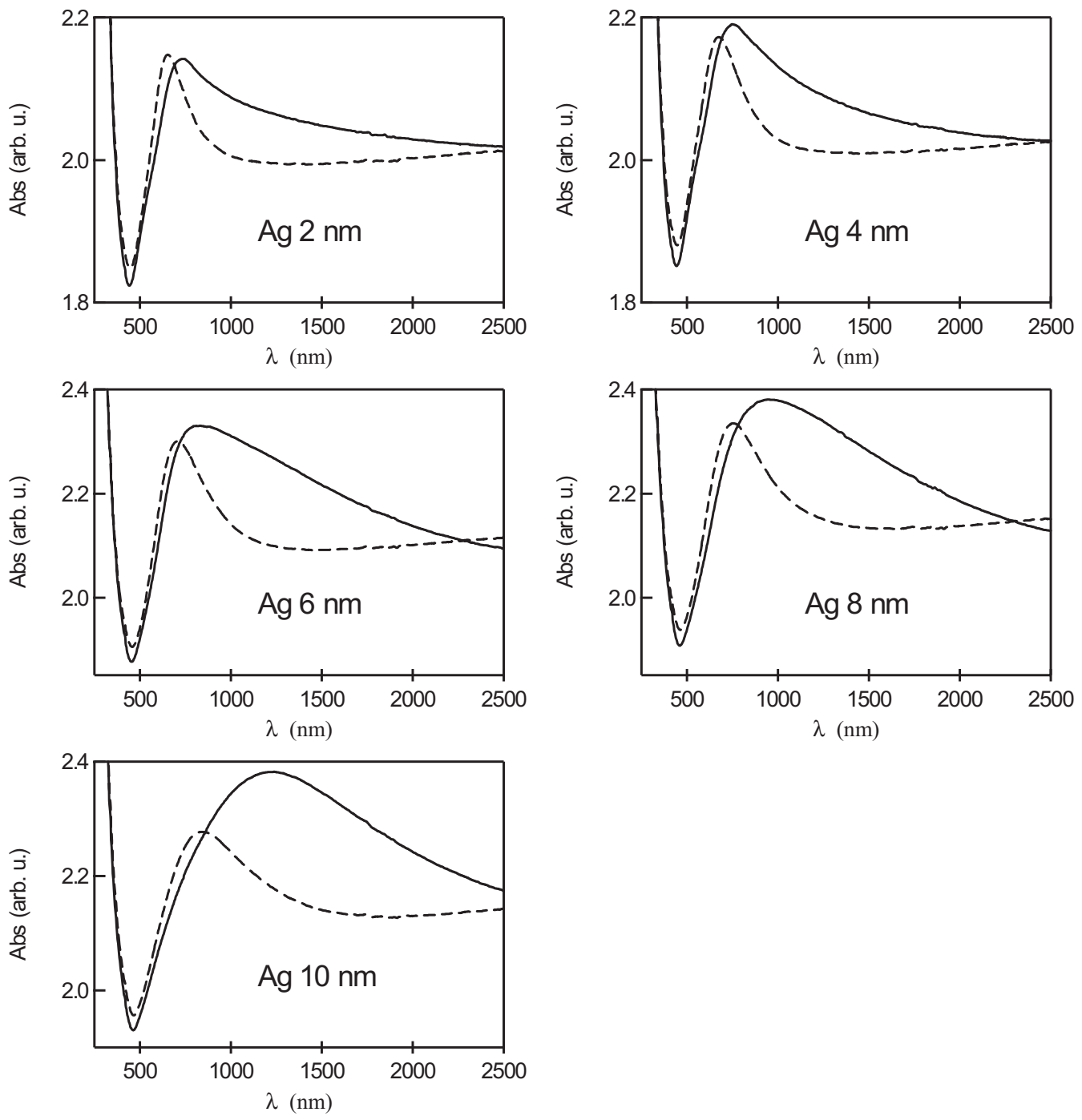

FIG. 4. Absorbance spectra of the TAVS samples with various Ag mass thicknesses for both of the semiconductor (solid curves) and the metal (dashed curves) phases.

dielectric function of $\mathrm{VO}_{2}$ exhibits the same spectral feature as what we observed in Fig. 1.

The phase transition of $\mathrm{VO}_{2}$ in the form of thin film is usually smeared out over a broad temperature interval rather than a sharp change at $68{ }^{\circ} \mathrm{C}$ for the bulk. ${ }^{28}$ Correspondingly, a gradual change in the optical constants (dielectric function or complex refractive index) of $\mathrm{VO}_{2}$ thin film was observed with increasing or decreasing temperature. ${ }^{29}$ Based on this fact a smart tuning of the temperature-dependent shift in $\lambda_{\text {SPR }}$ can be fulfilled. Figure 2(a) shows such a measurement for the AVS sample with $d_{m}=10 \mathrm{~nm}$. In this test the temperature was increased from 30 to $90{ }^{\circ} \mathrm{C}$ in a stepwise manner. In this case $\lambda_{\mathrm{SPR}}$ gradually blueshifts against temperature, as shown in Fig. 2(c). For comparison, a similar measurement was performed for the AQ sample also with $d_{m}=10 \mathrm{~nm}$. As expected, the LSPR peak in this case hardly shifts with temperature increasing. The results were plotted in Figs. 2(b) and 2(d).

We further found that the shift in $\lambda_{\mathrm{SPR}}$ is stable and reversible with correspondence to temperature from the measurement of absorbance spectrum with many repetitions of heating-cooling cycle, as shown in Fig. 3. For instance, re- peating the temperature cycle between 30 and $80{ }^{\circ} \mathrm{C}$ resulted in $\lambda_{\text {SPR }}$ shifting reversibly between 980 and $720 \mathrm{~nm}$. Such a stable and reversible behavior of LSPR is right in accordance with the phase transition characteristics of $\mathrm{VO}_{2}$ under temperature stimulation, ${ }^{18-20}$ suggesting again the origin of the thermal tunability of plasmon resonance of nano-Ag.

It has been demonstrated that the introduction of an external medium makes a dramatic effect on the resonance of Ag nanoparticles, ${ }^{11}$ other than the thermochromic material $\mathrm{VO}_{2}$ being discussed here. In order to gain more insight into the tunability, we used a dielectric layer $\mathrm{TiO}_{2}$ to overcoat the nano-Ag both for wavelength tuning and for protection of $\mathrm{Ag}$ surface from photochemical degradation caused by absorbed species. Figure 4 shows the absorbance spectra of the TAVS with various $\mathrm{Ag}$ mass thicknesses $\left(d_{m}=2,4,6,8\right.$, and 10 $\mathrm{nm})$. The solid curves show the results measured at $30{ }^{\circ} \mathrm{C}$, while the dashed curves at $80^{\circ} \mathrm{C}$ correspond to the semiconductor and metal phases of $\mathrm{VO}_{2}$, respectively. Similar to Fig. 1 , the resonance wavelengths $\lambda_{\text {SPR }}$ of the TAVS samples also shift to the blue when temperature is increased from 30 to $80{ }^{\circ} \mathrm{C}$, and the difference in $\lambda_{\mathrm{SPR}}$ between the two phases, $\Delta \lambda_{\text {SPR }}$, increases with Ag mass thickness. However, the ab- 


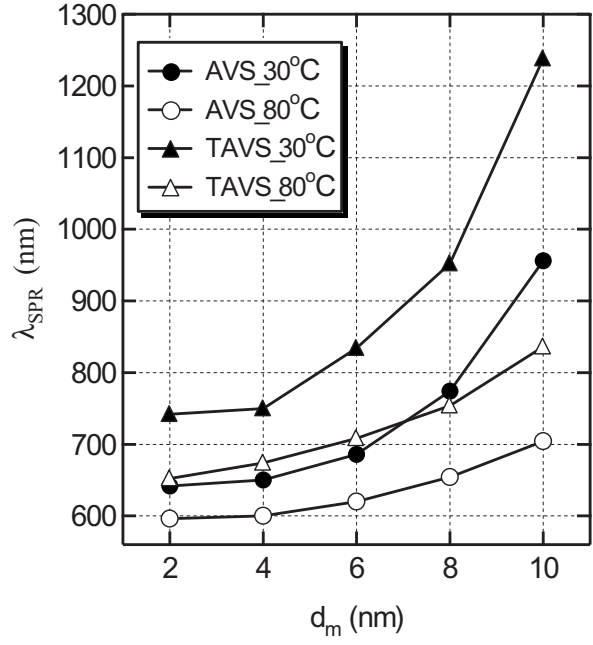

FIG. 5. Dependence of the resonance wavelength $\lambda_{\text {SPR }}$ on Ag mass thickness $d_{m}$ for the two series of samples, AVS and TAVS, measured at 30 and $80{ }^{\circ} \mathrm{C}$, respectively.

sorbance subpeak at $390 \mathrm{~nm}$ disappears because the strong interband absorption of $\mathrm{TiO}_{2}$ occurs at $\lambda<400 \mathrm{~nm}$ and covers up the relatively weak absorption from $\mathrm{VO}_{2}$.

Alhough the major spectral features of the TAVS sample are similar to those of the AVS, the effects of $\mathrm{TiO}_{2}$ on the tuning of $\lambda_{\mathrm{SPR}}$ are more significant. In Fig. 5, we summarize the dependence of $\lambda_{\text {SPR }}$ on Ag mass thickness for the two series of samples based on Figs. 1 and 4. Note that at the same measuring temperature of 30 or $80^{\circ} \mathrm{C}$, the curves for the TAVS sample lie above those for the AVS, suggesting an extra redshift in $\lambda_{\mathrm{SPR}}$ caused by the addition of $\mathrm{TiO}_{2}$. Further, we surprisingly find that $\Delta \lambda_{\text {SPR }}$ for the TAVS sample with $d_{m}=10 \mathrm{~nm}$ reaches up to about $400 \mathrm{~nm}$. This value is much larger than the similar case for AVS, a value of about 250 $\mathrm{nm}$. In fact, the TAVS sample at each $d_{m}$ always has a larger value of $\Delta \lambda_{\mathrm{SPR}}$ than the AVS sample does, as shown in Fig. 5. These results demonstrate the positive effects of the addition of $\mathrm{TiO}_{2}$, which not only leads to a redshift in $\lambda_{\mathrm{SPR}}$ but also greatly enhances the wavelength tunability. The enhancement results from the increasing difference in dielectric functions, ${ }^{21}$ specifically their real parts, between the two phases of $\mathrm{VO}_{2}$ as wavelength increases.

The shift in $\lambda_{\text {SPR }}$ varying with the dielectric environment of the nanoparticles can be described in terms of sensitivity factor. ${ }^{30,31}$ This factor is defined as $\Delta \lambda_{\mathrm{SPR}} / \Delta n$, in which $\Delta \lambda_{\text {SPR }}$ is the resulting wavelength shift by the change in refractive index $\Delta n$ of the surroundings. If we assume a nanoparticle sitting on a substrate, the surrounding dielectric function $\varepsilon$ contains the two contributions from the substrate $\left(\varepsilon_{\text {sub }}\right)$ and the external medium $\left(\varepsilon_{\text {ext }}\right)$. In a simple manner, $\varepsilon=n^{2}=\left(\varepsilon_{\text {ext }}+\varepsilon_{\text {sub }}\right) / 2 \cdot{ }^{32-34}$ For our AVS samples, $\varepsilon_{\text {ext }}$ is unity because the external medium is air and hence $\varepsilon=(1$ $\left.+\varepsilon_{\mathrm{VO}_{2}}\right) / 2$, in which $\varepsilon_{\mathrm{VO}_{2}}$ is the dielectric function of $\mathrm{VO}_{2}$. When the external medium of $\mathrm{TiO}_{2}$ was added to form the TAVS samples, thus $\varepsilon=\left(5.76+\varepsilon_{\mathrm{VO}_{2}}\right) / 2$, the dielectric constant of $\mathrm{TiO}_{2}$ is taken into account. Based on these definitions, the sensitivity factors for the two phases of $\mathrm{VO}_{2}$ can be calculated. The results were plotted in Fig. 6. As seen in the figure, the sensitivity factor generally increases with Ag mass

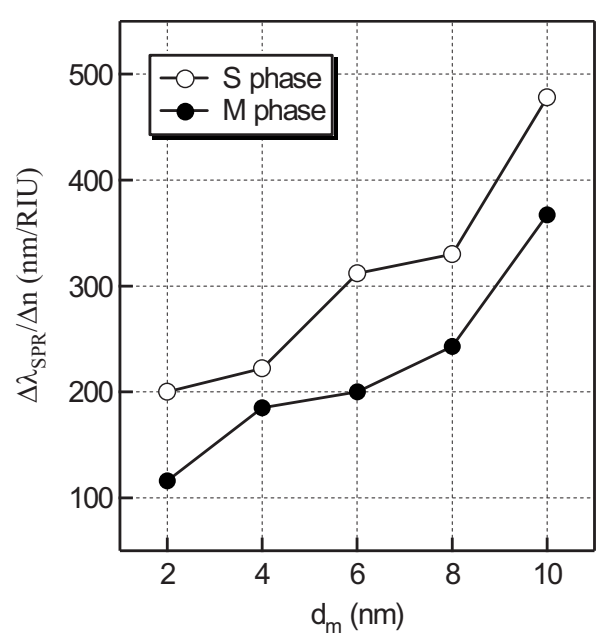

FIG. 6. Sensitivity factor as a function of Ag mass thickness $d_{m}$ for the AVS sample at the semiconductor (s) and metal (m) phases.

thickness $d_{m}$ for both of the two phases of $\mathrm{VO}_{2}$ and a value, as large as $478 \mathrm{~nm} /$ refractive index unit, was obtained for the semiconductor phase at $d_{m}=10 \mathrm{~nm}$. Such a large value means that the AVS sample is highly sensitive to the dielectric environment and may be used in nanoscale optical sensors.

\section{CONCLUSIONS}

In conclusion, we have studied in detail the SPR tunability of Ag nanoparticles on a thermochromic thin film of $\mathrm{VO}_{2}$. The resonance can be tuned thermally and is reversibly tunable in a wide spectral region due to the temperaturedependent dielectric properties of $\mathrm{VO}_{2}$. By controlling the temperature in a stepwise manner, the resonance wavelength can be fine tuned. Larger Ag nanoparticles or particle films with larger mass thickness make the tuning more pronounced and effective. The wavelength tunability can be further improved by combining $\mathrm{TiO}_{2}$ and $\mathrm{VO}_{2}$ as the surrounding dielectric medium of the nanoparticles. Additionally, it is demonstrated that $\mathrm{Ag}$ nanoparticles on $\mathrm{VO}_{2}$ possess a very high sensitivity to the external dielectric environment surrounding the particles. These results are expected to stimulate extensive research pertinent to the nanometal- $\mathrm{VO}_{2}$ composite systems for their potential usage in temperature-sensitive nanophotonics, nanosensor, and so on.

Our study actually opens a new way to manipulate the LSPR of meal nanoparticles characterized by large tunable wavelength range, thermal reversibility, and capability of fine tuning. Note that such tuning is activated by the change in refractive index of $\mathrm{VO}_{2}$ with temperature. We can modify the thermochromism of $\mathrm{VO}_{2}$ or use other thermochromic materials to tune the resonance for a wider spectral response or at a different temperature region.

\section{ACKNOWLEDGMENTS}

This work was supported by the National High Technology Research and Development Program of China (Grant Nos. 2007AA03Z326 and 2007AA05Z431) and the Bureau of Science and Technology of Guangzhou Municipality (Grant No. 2007Z2-D2051). 
${ }^{1}$ U. Kreibig and M. Vollmer, Optical Properties of Metal Clusters (Springer, Berlin, 1995).

${ }^{2}$ K. L. Kelly, E. Coronado, L. L. Zhao, and G. C. Schatz, J. Phys. Chem. B 107, 668 (2003).

${ }^{3}$ W. A. Murray and W. L. Barnes, Adv. Mater. (Weinheim, Ger.) 19, 3771 (2007).

${ }^{4}$ W. L. Barnes, A. Dereux, and T. W. Ebbesen, Nature (London) 424, 824 (2003).

${ }^{5}$ A. J. Haes and R. P. Van Duyne, J. Am. Chem. Soc. 124, 10596 (2002).

${ }^{6}$ J. Homola, Chem. Rev. (Washington, D.C.) 108, 462 (2008).

${ }^{7}$ C. L. Haynes and R. P. Van Duyne, J. Phys. Chem. B 107, 7426 (2003).

${ }^{8}$ R. A. Alvarez-Puebla, D. J. Ross, G. A. Nazri, and R. F. Aroca, Langmuir 21, 10504 (2005)

${ }^{9}$ T. R. Jensen, M. D. Malinsky, C. L. Haynes, and R. P. Van Duyne, J. Phys. Chem. B 104, 10549 (2000).

${ }^{10}$ M. Hirai and A. Kumar, J. Appl. Phys. 100, 014309 (2006).

${ }^{11}$ G. Xu, M. Tazawa, P. Jin, S. Nakao, and K. Yoshimura, Appl. Phys. Lett. 82, 3811 (2003).

${ }^{12}$ Y. T. Lim, J. K. Kim, Y. B. Shin, and B. H. Chung, Adv. Funct. Mater. 16, 1015 (2006).

${ }^{13}$ R. H. Doremus, J. Chem. Phys. 42, 414 (1965).

${ }^{14}$ U. Kreibig, J. Phys. F: Met. Phys. 4, 999 (1974).

${ }^{15}$ S. Link and M. A. El-Sayed, J. Phys. Chem. B 103, 4212 (1999).

${ }^{16}$ S. Link and M. A. El-Sayed, J. Phys. Chem. B 103, 8410 (1999).

${ }^{17}$ K. Watanabe, D. Menzel, N. Nilius, and H. J. Freund, Chem. Rev. (Washington, D.C.) 106, 4301 (2006).

${ }^{18}$ A. Zylbersztejn and N. F. Mott, Phys. Rev. B 11, 4383 (1975).

${ }^{19}$ M. Imada, A. Fujimori, and Y. Tokura, Rev. Mod. Phys. 70, 1039 (1998).
${ }^{20}$ T. D. Manning, I. P. Parkin, M. E. Pemble, D. Sheel, and D. Vernardon, Chem. Mater. 16, 744 (2004).

${ }^{21}$ G. Xu, Y. Chen, M. Tazawa, and P. Jin, J. Phys. Chem. B 110, 2051 (2006).

${ }^{22}$ P. Jin, M. Tazawa, and G. Xu, J. Appl. Phys. 99, 096106 (2006).

${ }^{23}$ M. Maaza, O. Nemraoui, C. Sella, A. C. Beye, and B. Baruch-Barak, Opt. Commun. 254, 188 (2005).

${ }^{24}$ M. Maaza, O. Nemraoui, C. Sella, and A. C. Beye, Gold Bull. 38, 100 (2005).

${ }^{25}$ M. B. Cortie, A. Dowd, N. Harris, and M. J. Ford, Phys. Rev. B 75, 113405 (2007).

${ }^{26}$ G. Xu, C. M. Huang, M. Tazawa, P. Jin, and D. M. Chen, J. Appl. Phys. 104, 053101 (2008).

${ }^{27}$ G. Xu, Y. Chen, M. Tazawa, and P. Jin, Appl. Phys. Lett. 88, 043114 (2006).

${ }^{28}$ G. I. Petrov, V. V. Yakovlev, and J. Squier, Appl. Phys. Lett. 81, 1023 (2002).

${ }^{29}$ H. Kakiuchida, P. Jin, S. Nakao, and M. Tazawa, Jpn. J. Appl. Phys., Part 2 46, L113 (2007).

${ }^{30}$ M. Duval Malinsky, K. L. Kelly, G. C. Schatz, and R. P. Van Duyne, J. Phys. Chem. B 105, 2343 (2001).

${ }^{31}$ G. Xu, M. Tazawa, P. Jin, and S. Nakao, Appl. Phys. A: Mater. Sci. Process. 80, 1535 (2005).

${ }^{32}$ C. G. Granqvist and O. Hunderi, Phys. Rev. B 16, 3513 (1977).

${ }^{33}$ R. Monique and R. Georges, J. Opt. Soc. Am. 67, 510 (1977).

${ }^{34}$ S. Yoshida, T. Yamaguchi, and A. Kinbara, J. Opt. Soc. Am. 62, 1415 (1972). 